\title{
STREAMLINE MODELING
}

\section{Designing Free-Form Surfaces By Blending Tangent Vectors}

\author{
Kenjiro T. Miura \\ Shizuoka University, Shizuoka, Japan \\ ktmiura@eng.shizuoka.ac.jp
}

Naoki Sakiyama

Shizuoka University, Shizuoka, Japan

s0830060@eng.shizuoka.ac.jp

Toru Kaneko

Shizuoka University, Shizuoka, Japan

kaneko@eng.shizuoka.ac.jp

\begin{abstract}
This paper proposes a new free-form surface design methodology named Streamline Modeling. The popular conventional surface construction method is to generate free-form surfaces by determining positions of their control points for Bézier, NURBS and other free-form surface formulations. They adopt a design strategy to use various blending functions to mix their control points' positions to define their shapes. Although they have nice geometric characteristics such as the convex hull and variation diminishing properties and they are frequently used in CAD/CAM as well as CG fields, they have potential instability of their curvature and variation of curvatures. Therefore in the Streamline Modeling, instead of blending positions, we adopt a design strategy to blend directions of given tangent vectors to define fair curves called streamlines and furthermore to blend the tangent vectors of these streamlines to generate isoparametric lines of a free-form surface. In this paper, we use unit quaternion curves in integral forms to blend tangent vectors to generate streamlines and isoparametic lines of surfaces as well. This enables us to control their curvature and variation of curvature more than is possible with traditional parametric representations.

The Streamline Modeling balances the efficiency of the design process and the fairness of the designed surfaces. The quality of the surface
\end{abstract}


examples shown in this paper is acceptable for industrial applications as well as CG animation.

Keywords: Streamline modeling, free-form surface

\section{Introduction}

Bézier and NURBS(Non-Uniform Rational B-Spline) surfaces are those of the most popular surface representations due to their nice geometric characteristics such as convex hull and variation diminishing properties [1]. The main idea of their surface construction shceme is to blend positions of points called control ones to define surface shapes.

However they have potential instability of their curvature and variation of curvatures: their curvature and variation of curvatures may change dramatically by repositioning their control points and have very complicated curvature or variation of curvature profiles because they are not parametrized by arc length and the curvature formulation have a square root term of polynomial functions as a denominator even though they are polynomial surfaces. We should investigate methods to use the arc length parametrization and to control tangent vectors directly. For a two-dimensional curve, a method that more directly control the tangent vector is the clothoid construction $[2,3,4]$. The clothoid, or Cornu spiral, is a plane curve defined parametrically in terms of Fresnel integrals as

$$
\left(\begin{array}{c}
x(u) \\
y(u)
\end{array}\right)=\pi B\left(\begin{array}{c}
C_{s}(u) \\
S_{n}(u)
\end{array}\right)
$$

where the scaling factor $\pi B$ is positive and the Fresnel integrals are

$$
\begin{aligned}
C_{s}(u) & =\int_{0}^{u} \cos \frac{\pi u^{2}}{2} d u, \\
S_{n}(u) & =\int_{0}^{u} \sin \frac{\pi u^{2}}{2} d u .
\end{aligned}
$$

The main objective of the clothoid construction is to specify its constant length tangent in terms of the trigonometric functions of a parameter proportional to the arc length, although this makes integration necessary to obtain its final shape. Since parameter $t$ is proportional to the arc length, the curvature and variation of curvature of the clothoid are given by simple functions of $t$-a linear function and a constant. Makino and et al.[5] developed a method to generate piecewise clothoid segments connected with $G^{2}$ continuity for specified connection points and tangent vectors there for the trajectory design of robot manipulators to enable 
smooth acceleration changes. Their method can be interpreted to blend tangent vectors by a quadratic functions in a two-dimensional plane.

In this paper, we have developed a new method to blend tangent vectors in three-dimensional space and proposes Streamline Modeling methodology for free-form surafce design where stremalines are generated based on our method by specifiying their tangent vectors and a surfaces is constructed as a set of these streamlines by blending their tangent vectors instead of their geometrical positions.

\section{BASIC CONCEPT OF STREAMLINE MODELING}

We would like to construct surfaces as sets of isoparametric lines called streamlines as shown in Figure 1. Assume that a surface is given by $S(s, t)$. Each streamline has a constant parameter value $t_{0}$ and starts from a point on $S\left(s_{0}, t_{0}\right)$ in the positive or negative $s$ direction. As shown in 1(a) $s_{0}$ may be equal to the minimum value $t_{\min }$ or the maximum value $t_{\text {max }}$ of $t$, or another value as shown in 1(b).

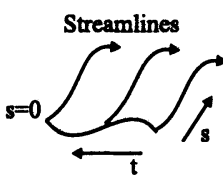

(a)

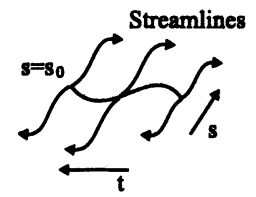

(b)

Figure 1 Streamlines as isoparametric lines

We prefer to specify the first derivative $\partial \boldsymbol{S}(s, t) / \partial s=s(s, t)$ with respect to the arc length parameter $s$ as streamlines. If it is given, $\boldsymbol{S}(s, t)$ can be defined by

$$
\boldsymbol{S}(s, t)=\boldsymbol{S}\left(s_{0}, t\right)+\int_{s_{0}}^{s} s\left(s, t_{0}\right) d s .
$$

If $s<s_{0}$, the above equation should be interpreted as

$$
\boldsymbol{S}(s, t)=\boldsymbol{S}\left(s_{0}, t\right)-\int_{s}^{s_{0}} \boldsymbol{s}\left(s, t_{0}\right) d s .
$$

Equation (3) tells us that by giving an isoparametric line at $s=s_{0}$ in parameter $t$ direction and the first derivative $s(s, t)$ in parameter $s$ direction over the whole parameter space, we can construct a surface. Since parameter $s$ is the arc length, the length of each streamline depends only on the maximum value of $s$ and we can make them dependent on $t$. 
From Equation (3) the first derivative of $S(s, t)$ with respect $t$, i.e. $\partial S(s, t) / \partial t=t(s, t)$ is given by

$$
\begin{aligned}
t(s, t) & =\frac{\partial \boldsymbol{S}(s, t)}{\partial t} \\
& =t_{0}+\int_{s_{0}}^{s} \frac{\partial s(s, t)}{\partial t} d s
\end{aligned}
$$

where $\boldsymbol{t}_{0}=\partial \boldsymbol{S}\left(s_{0}, 0\right) / \partial t$. Note that even though paramter $s$ is the arc length, $t$ is not because the norm of $t(s, t)$ is not guaranteed to be equal to 1 .

\section{B-SPLINE QUATERNION CURVE}

Kim et al. [7] proposed three types of quaternion curves-Bézier, Hermite, and B-spline. In this subsection, we review the B-spline quaternion curve, since we use it for our new curve and surface in this paper. An $k$ th order B-spline curve $\boldsymbol{C}(u)$ is defined with the B-spline basis functions $B_{i, k}(u)$, which are defined by the following recurrence relation [8]:

$$
B_{i, 1}(u)= \begin{cases}1 & \text { if } u_{i}<u<u_{i+1} \\ 0 & \text { otherwise }\end{cases}
$$

and

$$
\begin{aligned}
B_{i, k}(u)= & \frac{u-u_{i}}{u_{i+k-1}-u_{i}} B_{i, k-1}(u) \\
& \quad+\frac{u_{i+k}-u}{u_{i+k}-u_{i+1}} B_{i+1, k-1}(u) .
\end{aligned}
$$

For given control points $\boldsymbol{P}_{i}, i=0, \ldots, n$, the B-spline curve can be reformulated in the following cumulative form:

$$
\boldsymbol{C}(u)=\boldsymbol{P}_{0}+\sum_{i=1}^{n} \Delta \boldsymbol{P}_{i} \tilde{B}_{i, k}(u),
$$

where

$$
\Delta \boldsymbol{P}_{i}=\boldsymbol{P}_{i}-\boldsymbol{P}_{i-1}
$$

and

$$
\begin{aligned}
\tilde{B}_{i, k}(u) & =\sum_{j=1}^{n} B_{j, k}(u) \\
& = \begin{cases}\sum_{j=i}^{i+k-1} B_{j, k}(u) & \text { if } u_{i}<u<u_{i+k-1} \\
1 & \text { if } u \geq u_{i+k-1} \\
0 & \text { if } u \leq u_{i}\end{cases}
\end{aligned}
$$


By converting $\boldsymbol{C}(u)$ to $q(u), \boldsymbol{P}_{0}$ to $q_{0}, \Delta \boldsymbol{P}_{i}$ to $\omega_{i}$, and summation to quaternion multiplication, the corresponding quaternion curve is obtained in a cumulative form as follows:

$$
q(u)=q_{0} \prod_{i=1}^{n} \exp \left(\omega_{i} \tilde{B}_{i, k}(u)\right)
$$

where

$$
\omega_{i}=\log \left(q_{i-1}^{-1} q_{i}\right) .
$$

This B-spline quaternion curve is $C^{k-2}$-continuous and locally controllable with the control quaternions $\left\{q_{i}\right\}$ and the angular velocities $\left\{\omega_{i}\right\}$.

Note that the above formulation is using the local frame. If the global frame is adopted, the sequence of the rotations of Equation (12) is reversed and the equation is reformulated by

$$
q(u)=\left(\prod_{i=n}^{1} \exp \left(\omega_{i} \tilde{B}_{i, k}(u)\right)\right) q_{0} .
$$

\section{BLENDING TANGENT VECTORS}

The unit quaternion curves proposed by Kim et al. [7] preserve most of the algebraic and differential properties of the original spline curves, and we chose them, especially the B-spline type, for blending tangent vectors.

However, the specifications of direction and orientation are not the same and an infinite number of rotations is possible to change one direction to another. This suggests that we must reconsider how to determine $\omega_{i}$ in Equation (14). Tangent vectors $\boldsymbol{t}_{i}$ instead of $q_{i}, i=0, \ldots, n$ are preferable for our purposes to define a unit quaternion curve because $q_{i}$ is not unique for its corresponding $\boldsymbol{t}_{i}$.

If the length of a streamline is $l, s$ varies from 0 to $l$ and it differs from the parameter $u$ of a B-spline curve, whose parameter can be normalized $(0 \leq u \leq 1)$ by changing its knot vector. Hence assume $u=s / l$, Equation (14) becomes

$$
q(u)=q\left(\frac{s}{l}\right)=\left(\prod_{i=n}^{1} \exp \left(\omega_{i} \tilde{B}_{i, k}\left(\frac{s}{l}\right)\right)\right) q_{0}, \quad 0 \leq u \leq 1 .
$$

We can now use the B-spline unit quaternion curve $q(u)$ to define the tangent vector of the streamline $s(s)$ as follows:

$$
\boldsymbol{s}(s)=q(u) \hat{\boldsymbol{v}}_{0} q(u)^{-1}
$$


where $\hat{\boldsymbol{v}}_{0}$ is an arbitrary unit vector.

Here is a simple interpolation problem. Find a fair curve whose tangent vectors at its initial and end points are given. One possible answer, preferable in our sense of fairness, is a circular arc, because if we rotate its tangent with a constant angular velocity, the resulting curve is a circular arc, which has a constant curvature.

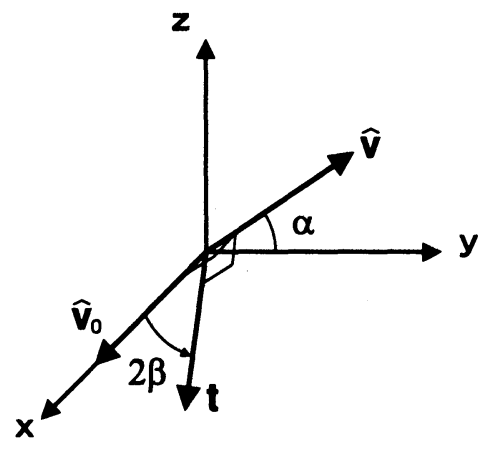

Figure $2 q=(\cos \beta, 0, \cos \alpha \sin \beta, \sin \alpha \sin \beta)$ for a given $t$

For the given two unit tangent vectors $\boldsymbol{t}_{0}=q_{0} \hat{\boldsymbol{v}}_{0} q_{0}^{-1}$ and $\boldsymbol{t}_{1}=q_{1} \hat{\boldsymbol{v}}_{0} q_{1}^{-11}$, the geodesic curve $\gamma_{t_{0}, t_{1}}(u)$ in $S^{2}$, connecting the end points of two unit tangent vectors $t_{0}$ and $t_{1}$ is a great arc between the two end points and is given by

$$
\gamma_{t_{0}, t_{1}}(u)=q_{t_{0}, t_{1}}(u) t_{0} q_{t_{0}, t_{1}}^{-1}(u)
$$

where

$$
q_{t_{0}, t_{1}}(u)=\exp \left(u \cdot \omega_{t_{0}, t_{1}}\right) .
$$

$\omega_{t_{0}, t_{1}}$ is an angular velocity of the rotation, and its direction is equal to a cross product $\left[t_{0}, t_{1}\right]$ of $t_{0}$ and $t_{1}$, as shown in Figure 3 . It is given by

$$
\begin{aligned}
\omega_{t_{0}, t_{1}} & =\frac{1}{2} \sin ^{-1}\left(\left|\left[t_{0}, t_{1}\right]\right|\right) \frac{\left[\boldsymbol{t}_{0}, \boldsymbol{t}_{1}\right]}{\left|\left[\boldsymbol{t}_{0}, \boldsymbol{t}_{1}\right]\right|} \\
& =\frac{1}{2} \theta_{t_{0}, t_{1}} \hat{\boldsymbol{v}}_{t_{0}, t_{1}},
\end{aligned}
$$

where $\theta_{t_{0}, t_{1}}$ is the angle between $t_{0}$ and $t_{1}$ and $\hat{v}_{t_{0}, t_{1}}$ is a unit vector whose direction is equal to $\left[t_{0}, t_{1}\right]$.

The above $\omega_{t_{0}, t_{1}}$ makes $\gamma_{t_{0}, t_{1}}(u)$ satisfy $\gamma_{t_{0}, t_{1}}(0)=t_{0}$ and $\gamma_{t_{0}, t_{1}}(1)=$ $t_{1}$. The resulting curve, whose integrand is $q_{t_{0}, t_{1}}(u) t_{0} q_{t_{0}, t_{1}}^{-1}(u)$, is a circular arc. Furthermore, $\omega_{t_{0}, t_{1}}$ depends on only $t_{0}$ and $t_{1}$ and does not 


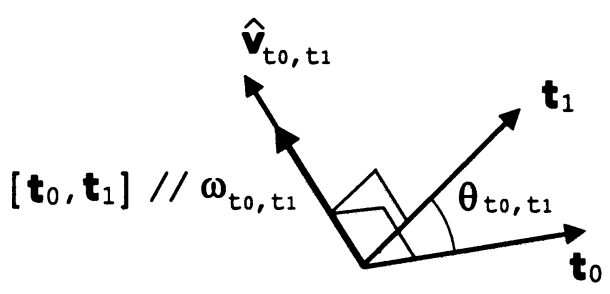

Figure 3 The axis of the rotation

depend on the choices of $q_{0}$ or $q_{1}$ that are not unique for $t_{0}$ and $t_{1}$. If we use $\omega_{t_{0}, t_{1}}=\log \left(q_{0}^{-1} q_{1}\right)$ (similar to Equation (13)), the resulting curve is generally not a circular arc.

\subsection{BLENDING TANGENT VECTORS BY B-SPLINE UNIT QUATERNION CURVE}

Based on the discussion in the previous subsection, for Equation (14), we use $\omega_{i}$ given by

$$
\omega_{i}=\frac{1}{2} \sin ^{-1}\left(\left|\left[t_{i-1}, t_{i}\right]\right|\right) \frac{\left[t_{i-1}, t_{i}\right]}{\left|\left[t_{i-1}, t_{i}\right]\right|}
$$

instead of $\log \left(q_{i-1}^{-1} q_{i}\right)$ to define our new B-spline unit quaternion curve. The above $\omega_{i}$ makes sense in the global frame because it is defined by vectors in the global frame. This is the reason why we adopted the global frame instead of the local one.

Figure 4 shows differences between curves generated on the unit sphere by Kim's original B-spline quaternion curve and our new one. The curves are traces of the end points of the tangent vectors controlled by the unit quaternion curves.

We uses the B-spline quaternion curve proposed here to blend the tangent vectors. Similarly, Bézier and Hermite unit quaternion curves can be defined based on their cumulative forms.

\section{EXAMPLES OF QI SURFACES}

In this section, we show several examples of QI surfaces in Figure 7-11 designed using our prototype system.

\subsection{DESIGN TOOL}

To design streamlines, we must specify several unit tangent vectors. Specifying a unit vector is equivalent to specifying a point on the Gaussian sphere, a unit sphere in 3D space. Our prototype system provides 


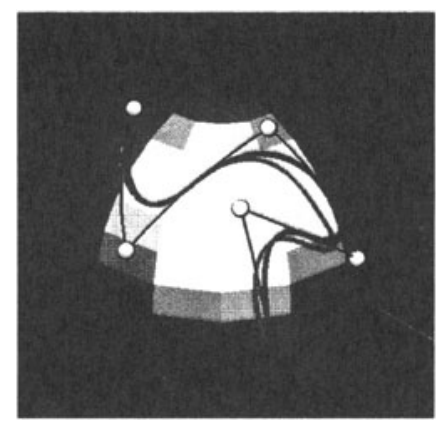

Figure 4 Comparison of two unit quaternion curves; Green curve: based on Kim's original (it depends on the choices of $q_{i}$ ), Red curve: our proposed one, Blue curve: great arcs between two end points

the users with a design tool to select a point on the sphere to specify a tangent vector. As shown in Figure 5, the users can pick the end point of a tangent vector on the sphere and drag it to an arbitrary position. Or, as shown in Figure 6, they can pick an arrow corresponding to a tangent vector and drag it. The arrows have specially shaped heads according to their index numbers; the users select one of them, even though several arrows are oriented in the same direction. To help the users know the property of the streamlines being designed, a curve traced by the end point of the tangent on the sphere is drawn with a color indicating the curvature of the streamline corresponding to the selected tangent vector sequence.

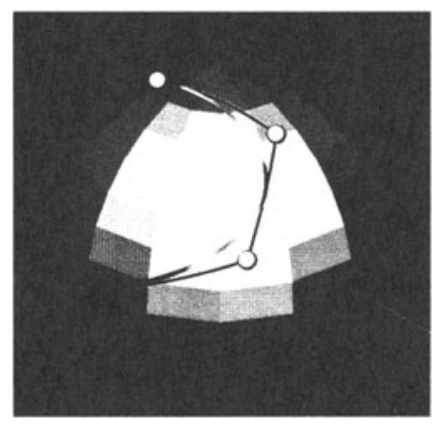

Figure 5 Points on the Gaussian sphere to specify tangent vectors. The curve on the sphere is a trace of the end points of the tangent vector; red color indicates high curvature and blue, low curvature of the corresponding streamline. The color changes from red, yellow, green, or cyan to blue according to the curvature. 


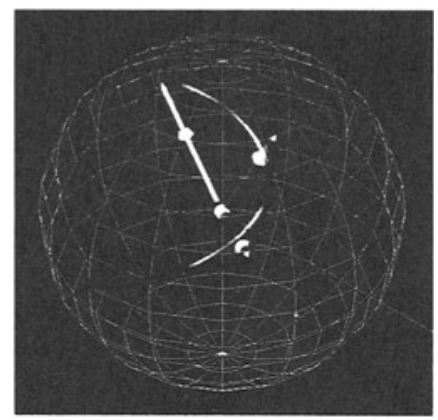

Figure 6 Arrows corresponding to tangent vectors

\subsection{INTEGRATION}

One drawback of a surface is that integration is necessary to obtain its shapes. Our prototype system performs integration using Simpson's method, a standard numerical integration method. Although we reported on hardware to accelerate integration for streamlines in another paper [10], changing the interval of Simpson's method enables the system to trade-off accuracy and integration speed. Initially, a larger interval is used to speed up the integration for better interactivity; in the final stage, a smaller interval is used to obtain an accurate shape.

For better interactivity once the integration has been performed, the calculated points are preserved and used for display until the curve or surface is modified. They are approximated by a B-spline curve or surface for display, as well as data transfer to other systems, as explained in the following subsection.

\subsection{APPROXIMATION BY B-SPLINE SURFACE}

B-spline surfaces are standard representations of free-form curves and surfaces and many CAGD, CAD/CAM, and CG systems use them as their data exchange formats. Our system can approximate QI surfaces by B-splines. The least square method is used for approximation to minimize the total value of square of the distance of the corresponding points of QI and B-spline surfaces. If the total value is larger than a given threshold, a new knot is inserted to the knot vector in each parameter direction. These processes are repeated until the total value becomes less than the threshold. 


\subsection{B-SPLINE QI SURFACES FOR INDUSTRIAL APPLICATIONS}

Several examples for industrial applications are given below. After designing key surfaces using the quadratic B-spline QI surface formulation ${ }^{2}$, each example is approximated by B-spline surfaces and transferred to commercial CAGD software. A total geometrical object is constructed to model a car or a ship based on these surfaces.

\section{CONCLUSIONS}

We proposed the Streamline Modeling based on our new method to blend directions of tangent vectors. In the Streamline Modeling, the designers can manipulate isoparametric lines of the surface called streamlines by specify their tangent vetors and the system blends these tangent vectors to generate the surface instead of blending their positions. The designers can pay attension to and manipulate intrinsic flows of surafce shapes by controling their streamlines.

The concept of the Streamline Modeling is quite different from traditional surface modeling strategies. Therefore in the future we will apply the methodology for various fields of geometric designs.

\section{Notes}

1. Note that for a given tangent vector $t, q$ does not uniquely satisfy $t=q \hat{v}_{0} q^{-1}$, as an infinite number of rotations is possible to change $\hat{v}_{0}$ to $t$. Assume $\hat{v}_{0}=(1,0,0), q$ is given by, for example, $\cos \beta+\hat{v} \sin \beta=(\cos \beta, 0, \cos \alpha \sin \beta, \sin \alpha \sin \beta)$ as shown in Figure 2 .

2. The length of an isoparametric line $l_{s}(t)$ of each B-spline QI surface is given by a quadratic function which satisfies $l_{s}(0)=l_{s}\left(l_{t}\right)=l_{s, \text { side }}$ and $l_{s}\left(l_{t} / 2\right)=l_{s, \text { center }}$.

\section{References}

[1] G. Farin, Curves and Surfaces for Computer Aided Geometric Design, Academic Press, 1988.

[2] E. Mehlum, "Nonlinear Splines," R.E. Barnhill, and R.F. Riesenfeld, ed., Computer Aided Geometric Design, Academic Press, pp.173-207, 1974.

[3] D.J. Walton, and D.S. Meek, "Clothoidal Splines," Computers and Graphics, vol.14, no.1, pp.95-100, 1990.

[4] D.S. Meek, and R.S.D. Thomas, "A Guided Clothoid Spline," Computer Aided Geometric Design, vol.8, pp.163-174, 1991.

[5] H. Makino, and H. Suda, "Continuous Path Control Using Clothoidal Interpolation," Proc. 20th ISIR, Tokyo, pp.1159, 1989. 


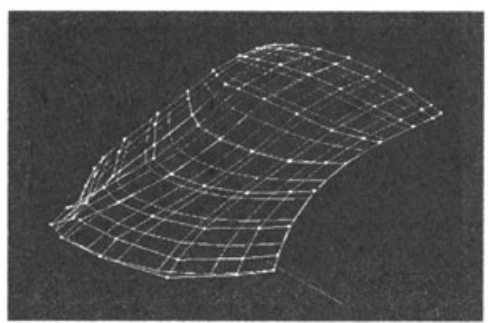

(a) Approximation of a QI surface by B-spline

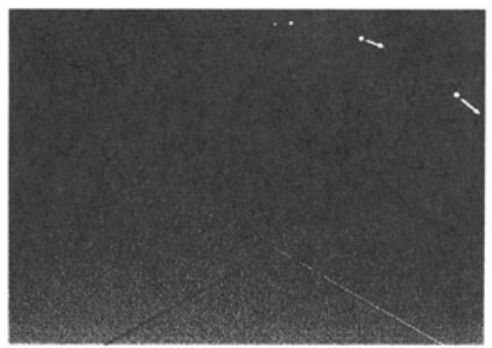

(c) Tangent vectors in $t$ direction

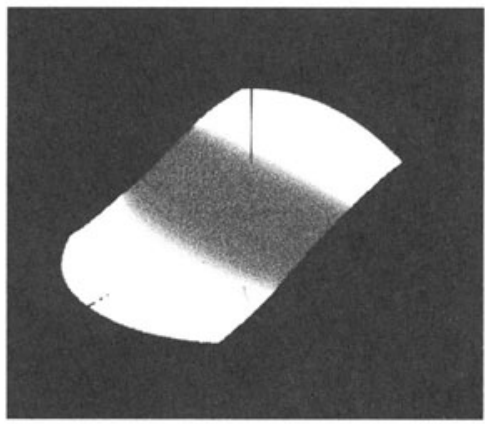

(e) Gaussian curvature

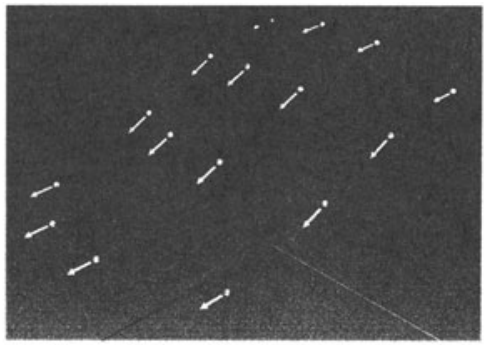

(b) Tangent vectors in $s$ direction

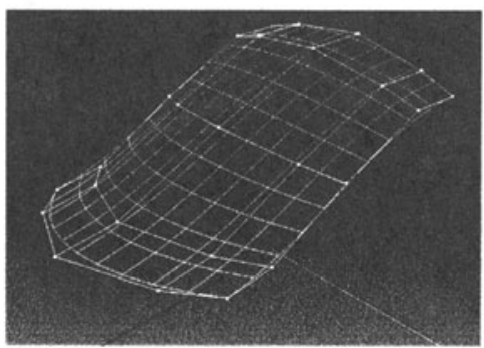

(d) B-spline approximation

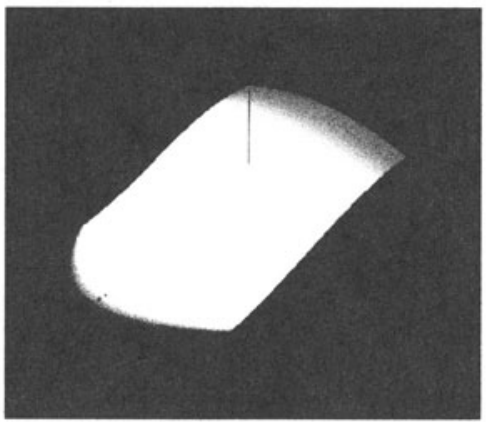

(f) Mean curvature

Figure 7 B-spline QI surface example: Car model No.1 


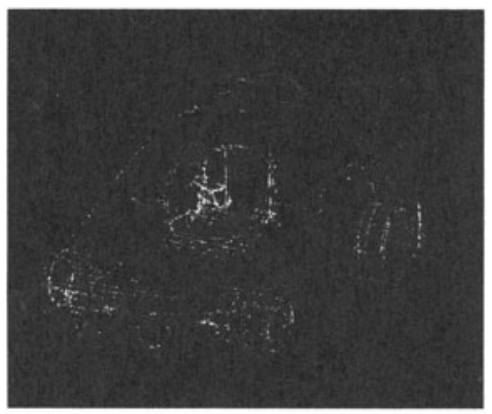

(a) Contour lines

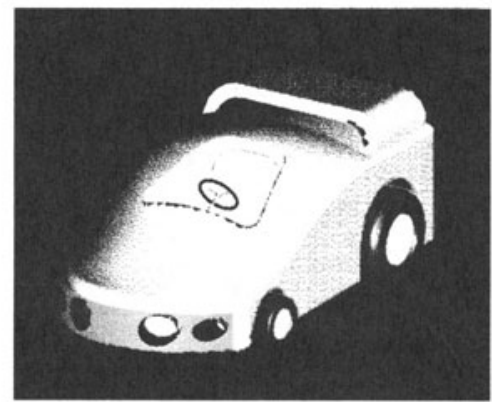

(b) Rendered model

Figure 8 B-spline QI surface example: Car model No.1

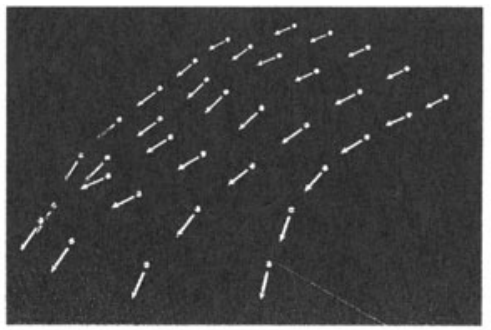

(a) Tangent vectors in $s$ direction

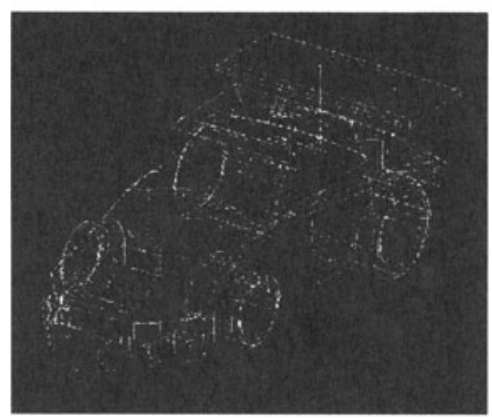

(b) Contour lines

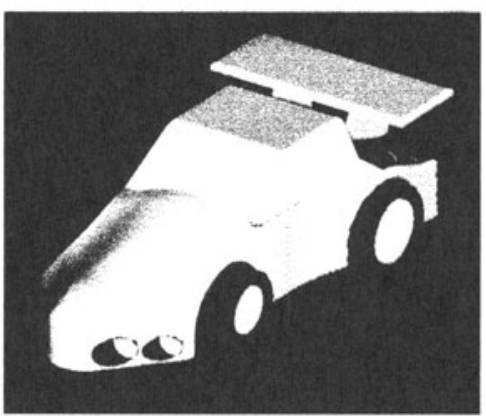

(c) Rendered model

Figure 9 B-spline QI surface example: Car model No.2 


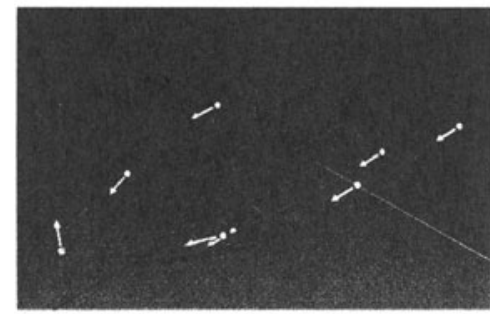

(a) Tangent vectors in $s$ direction for head of the ship

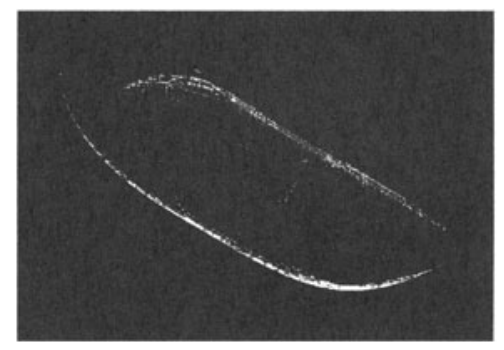

(c) Contour lines of the ship

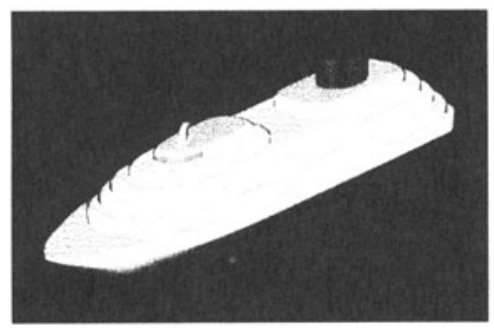

(e) Another view of the ship

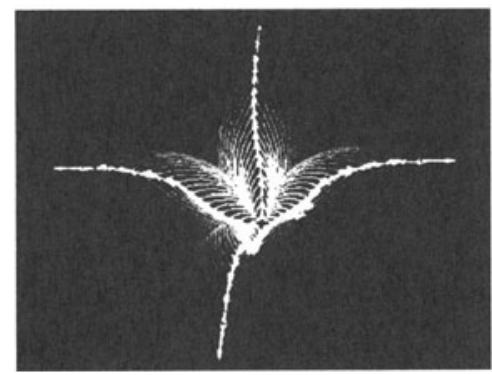

(g) Tangent vectors in $s$ direction of the flower

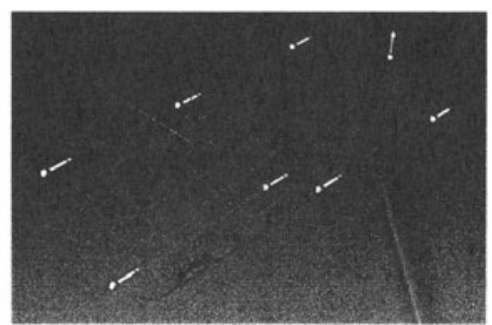

(b) Tangent vectors in $s$ direction for tail of the ship

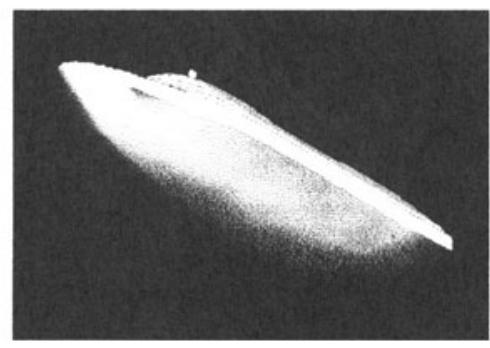

(d) Rendered model of the ship

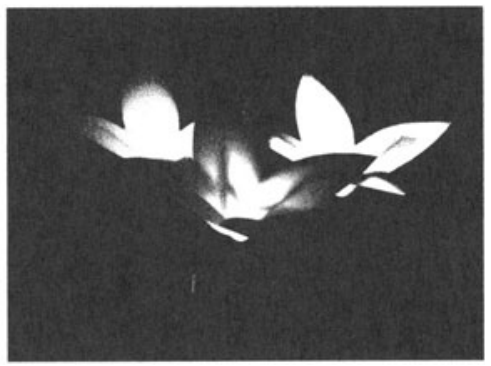

(f) Several flowers

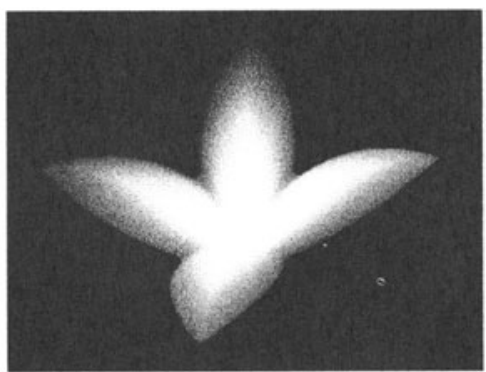

(h) Rendered model of the flower

Figure 10 B-spline QI surface example: Flower 
[6] M.P. do Carmo, Differential Geometry of Curves and Surfaces, Prentice Hall, 1976.

[7] M.-J. Kim, M.-S. Kim, and S.Y. Shin, "A General Construction Scheme for Unit Quaternion Curves with Simple High Order Derivatives," Computer Graphics(Proc. of SIGGRAPH '95), pp.369-376, 1995.

[8] C. De Boor, A Practical Guide to Splines, Springer-Verlag, 1978.

[9] I.L. Kantor, and A.S. Solodovnikov, Hypercomplex Numbers, Springer-Verlag, 1989.

[10] K.T. Miura, T. Nakaseko, and T. Ikedo, "A New Type of FreeForm Curve Given By An Integral Form ," CG International'98, Hannover, pp.722-725, 1998.

[11] L. Wang, X. Zhu and Z. Tang, "Coons type blended B-spline surface and its conversion to NURBS surface," Computer and Graphics, Vol. 21, No.3, pp.601-606, 1997. 